\title{
Evaluation of Heart Rate in Daily Life Based on 10 Million Samples Database
}

\author{
Makoto Komazawa ${ }^{1}$, Kenichi Itao ${ }^{1}$, Guillaume Lopez ${ }^{2} \&$ Zhiwei Luo ${ }^{3}$ \\ ${ }^{1}$ WINFrontier Laboratory, WINFrontier Co. Ltd., Tokyo, Japan \\ ${ }^{2}$ Faculty of Science and Engineering, Aoyama Gakuin University, Sagamihara, Japan \\ ${ }^{3}$ Organization of Advanced Science and Technology Kobe University, Kobe, Japan \\ Correspondence: Makoto Komazawa, WINFrontier Laboratory, WINFrontier Co. Ltd. 247 Shin-Yurakucho \\ Building, 1-12-1 Yurakucho, Chiyoda-ku, Tokyo, 100-0006 Japan.
}

Received: June 10, 2017 Accepted: July 10, 2017 Online Published: July 14, 2017

doi:10.5539/gjhs.v9n9p105 URL: https://doi.org/10.5539/gjhs.v9n9p105

\begin{abstract}
This research uses a very-large amount of heart rate data (approximately 10 millions entries) collected on one million persons to investigate heart rate trends in daily life. Trends were examined regarding both human characteristics (gender, age, BMI), and environmental properties (hour of the day, day of the week, season). Concerning the relation between heart rate and gender, women's heart rate trend was significantly lower compared to men's. As for the relation between heart rate and age, on one hand, women's heart rate tends to become lower with aging. On the other hand, men's heart rate tends to keep increasing until the 50's. Also, men's heart rate is lower than women's in the 10's and 20's, the trend becomes opposite from the 30's. In the relation between heart rate and BMI (Body Mass Index), heart rate is significantly higher for users with higher BMI. Regarding diurnal fluctuation of heart rate, it has been confirmed to take its lowest between 3 A.M. and 6 A.M., then increasing regularly until the peak between noon and 3 P.M., after which it decreases during the evening through the night. As for in-week fluctuation, the trend of higher heart rate during the week and lower heart rate during week-ends and holidays has been comfirmed. Specialy, heart rate remarkably increases from Sunday to Monday. Finally, concerning seasonal fluctuation trend, heart rate is decreasing from summer to autumn, and is at his highest level in winter. The results of this research tend to correspond with previous studies that have been carried out on smaller samples and fewer subjects, demonstrating the credibilitay and validity of the data measured by our system. Moreover, on a public health point of view, we can say that it is important to take into account various human properties and spatio-temporal characteristics when having a comparative analysis of people's health distribution. We think the knowledge obtained in this research can be applied practically to the whole society as an indication for health care and various diseases prevention.
\end{abstract}

Keywords: smartphone, large amount of measurement data, heart rate, daily life

\section{Introduction}

\subsection{Social and Technological Background}

Japan is entering a super aging society, the elderly who need medical care and nursing care rapidly increases, and the burden of medical care and nursing care expenses is expected to increase more and more. In addition, with the advancement of informatization, various stressors are increased along with the rapid changing lifestyle, and consequently results in an increasing number of people claiming a deterioration of their quality of life (QOL) and a collapse of the mind and body from fatigue and stress. According to the epidemiological survey conducted in 1999 by the Ministry of Health, Labor and Welfare (MHLW) division for Fatigue Research, the percentage of people who are aware of fatigue represents about $60 \%$ (47.2 million) of the working population, half (29.6 million) being shown to suffer from chronic fatigue lasting more than six months (Kitani, 200). According to statistics of the Ministry of Health, Labor and Welfare, from a study carried out in 2010 about the percentage of worries and stress in daily life, $46.5 \%$ of people are living with some stress (MHLW, 2010).

In such social environment, in the future, it can be said that not only to treat at a medical institution after suffering from a disease but also to grasp scientifically and manage their own health status daily, is very important for prevention. Though human vital signs include body temperature, blood pressure, heart rate (pulse rate), etc., heart 
rate is particularly an important indicator to grasp the state of the circulatory system, and it is said that symptoms of disease appear in heart rate (Yamashina, 2011). In addition, it is said that the average lifetime total heart rate of Japaneses, whose average life expectancy exceeds 80 years, is about 3 billion times, and cardiovascular aging and blood vessel disorder progresses at each heartbeat. If so, it can be said that it is important to accumulate heartbeats or carefully consider the heart rate every day (Yamashina, 2011).

In previous studies, there are several examples analyzing the relationship between heart rate, aging, BMI, and diurnal variation (Mason, 2007, Nakayama, 1999, Saito, 1998, Vandewale, 2007). However, in these previous studies, many are analyzing based on the heart rate at rest measured at a facility such as a medical institution, and there are only few studies based on the heart rate during everyday life tacking into account the influence of external factors such as mental stress due to work, physical activity, climate, etc. In addition, the target subjects number are small or medium, and there are no studies currently targeting large scale big data. Also, there are still few research examples that examined the trend of heart rate on long-term daily life such as weekly fluctuation of heart rate and seasonal fluctuation of heart rate.

\subsection{Purpose of this Research}

In a previous work, in order to collect a large amount of vital data, the authors have developed a system (COCOLOLO) that can analyze the heart rate variability from the color of the skin (change in brightness) by hitting the fingertips on the camera of a smartphone. Currently, this system is practically downloaded and used by about 1.3 million users (as of January 2017) practically as Apple Inc.'s iPhone application (WINFrontier, itunes) and Google Inc.'s Android application (WINFrontier, google play) (in Japanese). By using this system, it became possible to easily grasp heart rate variability and record data, anytime, anywhere, at any time in everyday life.

In this research, we analyze the trend of heart rate in daily life of people based on massive measurement data (about 10 million samples), collected using this smartphone application software. The following five items were analyzed and compared with the results measured by dedicated equipment in previous research, and the reliability and validity of the data measured in this system were investigated: heart rate and gender/age, heart rate and BMI, heart rate diurnal fluctuation, heart rate in-week fluctuation, heart rate seasonal fluctuation

\section{System Outline and Measured Data}

In this research, we used the above described heart rate variability analysis system that uses smartphones' camera (see Figure 1). This is a client/server system that consists of a smartphone, which is the client terminal, and a server that performs heart rate variability analysis processing. As humans heart beats, the amount of hemoglobin contained in the blood flow increases and decreases, so the apparent color of the skin at the fingertip changes slightly influenced by this phenomenon (Toritani, 2012). Therefore, by using a smartphone, a pulse wave waveform can be detected from the color (change in brightness) of the skin by applying a fingertip to the camera part, and the heart rate variation is analyzed from the fluctuations of the pulse wave.

The present system improves analysis accuracy by applying unique filtering method to remove noise, and as for the heart rate, compared with a system using a dedicated fingerpiece pulse wave sensor, it showds a correlation of 0.99 as shown in Figure 2, which can be assumed as almost the same accuracy 0.99 (Komazawa, 2016). By using this system, it became possible to measure heart rate variability easily, anytime, anywhere, at any occasion in daily life. In addition, by establishing the technology of collecting, storing, and processing health data, the user's vital data are transmitted to the server in real time, and it became possible to analyze and evaluate the heart rate at that time of daily life is measured based on large-scale measurement data. 


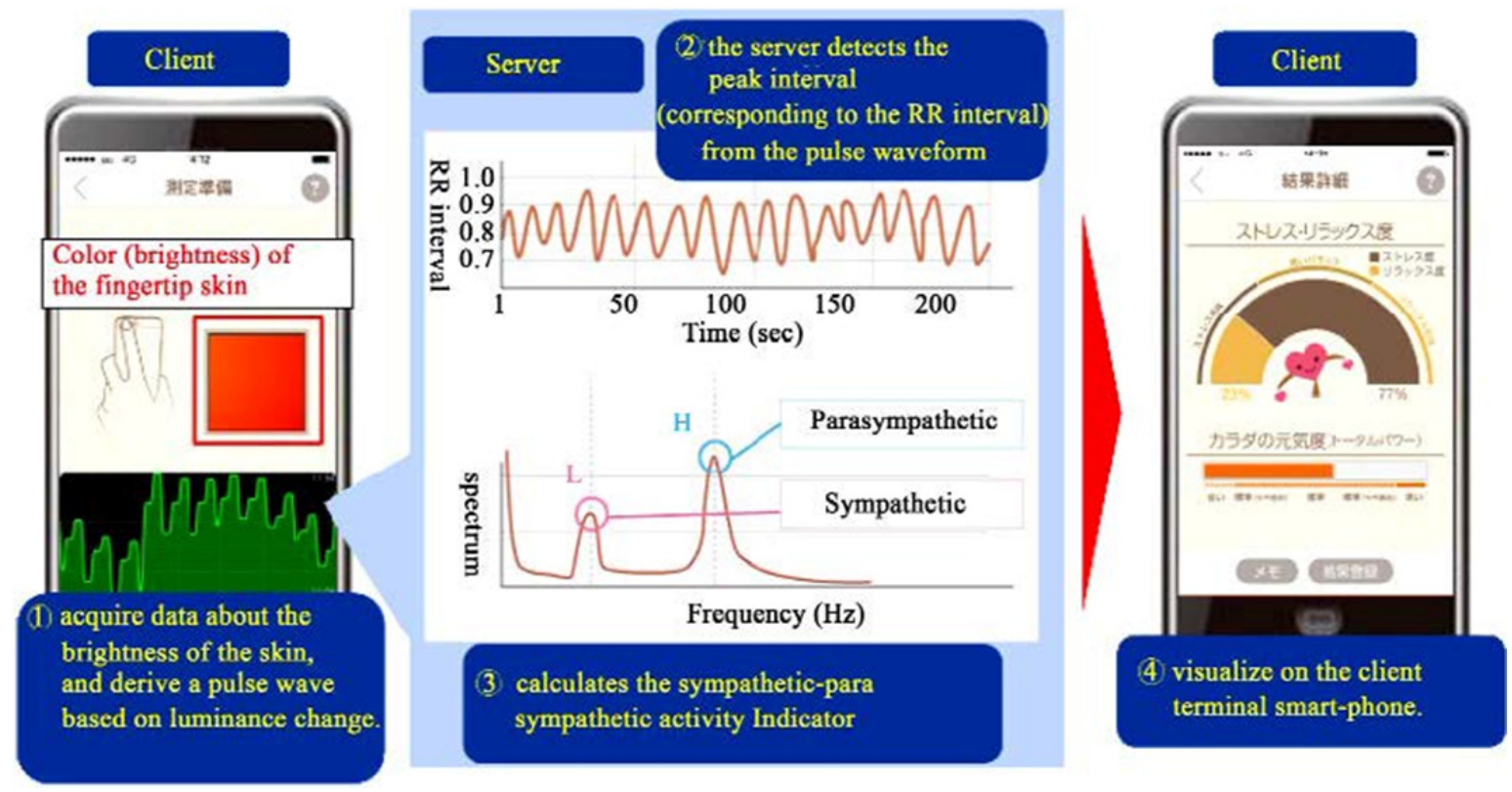

Figure 1. Smartphone application based measurement system outline

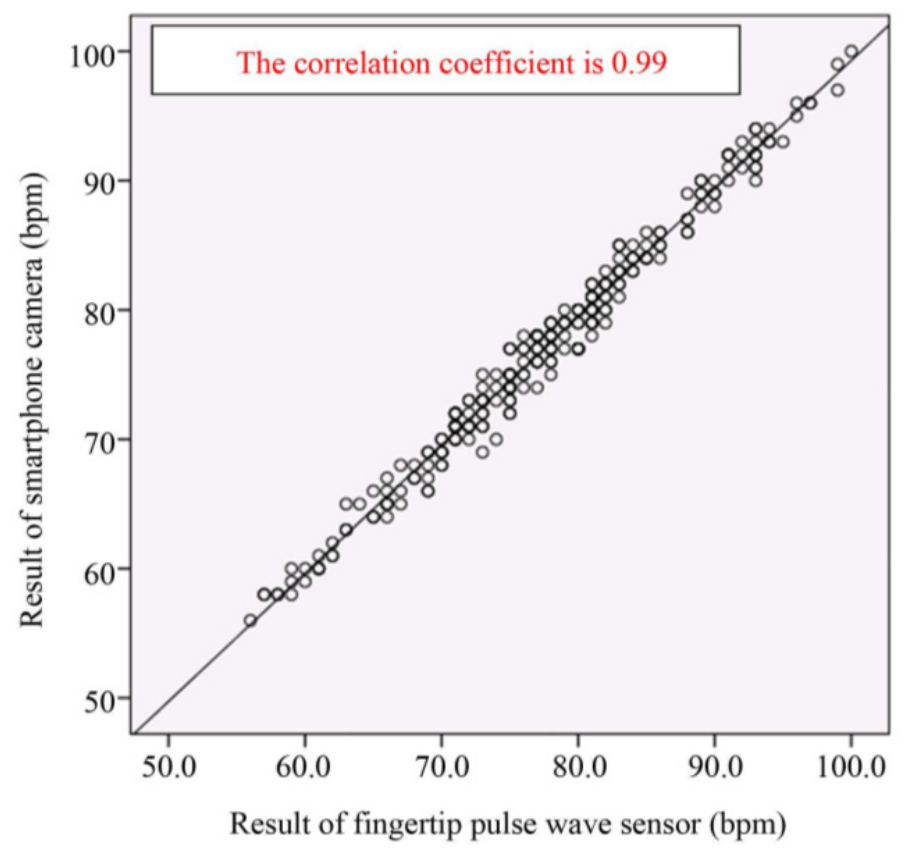

Figure 2. Correlation between heart rate measured by finger plethysmograph and heart rate estimated from smartphone camera (developed system)

In this study, 11443795 data (male: 3082010, female: 8361785) measured between February 2015 and February 2017 from 944775 persons (male: 260144, female: 684631) were analyzed based on big data of heart rate during daily life. Measurements with more than $20 \%$ of the pulse peak interval missing were excluded from the analysis targets. Regarding the use of measurement data, consent of the examiner is obtained based on the ethical regulations of WINFrontier Corporation. Table 1 shows the attributes of the users (left), and the attributes of measured data (right). We used IBM SPSS Statics Version 22 for statistical processing in this study. The significance level of the test was set at $5 \%$. 
Table 1. Characteristics of system's users (left) and measured data (right)

\begin{tabular}{cccc}
\hline Age & Man & Female & Total \\
\hline 10's & 34,439 & 98,208 & 132647 \\
20's & 68,097 & 220,821 & 288918 \\
30's & 47,365 & 123,664 & 171,029 \\
40's & 53,497 & 136,054 & 189,551 \\
50's & 41,271 & 88,911 & 130,182 \\
60's & 15,475 & 16,973 & 32,448 \\
Total & 260,144 & 684,631 & 944,775 \\
\hline
\end{tabular}

\begin{tabular}{cccc}
\hline Age & Man & Female & Total \\
\hline 10 's & 144,916 & 497,383 & 642,299 \\
20 's & 369,902 & $1,393,155$ & $1,763,057$ \\
30 's & 452,934 & $1,410,187$ & $1,863,121$ \\
40 's & 821,942 & $2,514,356$ & $3,336,298$ \\
50 's & 861,684 & $2,064,789$ & $2,296,473$ \\
60 's & 430,632 & 481,915 & 912,547 \\
Total & $3,082,010$ & $8,361,785$ & $11,443,795$ \\
\hline
\end{tabular}

\section{Analysis of Measured Data}

\subsection{Relation Between Heart Rate and Gender}

\subsubsection{Gender Differences in Heart Rate at Rest}

There is a gender difference in heart rate, and it has been reported that women's heart rate is generally higher than men's (Sagie, 1992, Singh, 1999). In a previous study, resting heart rate was analyzed from electrocardiogram of 46129 citizens who participated in a clinical trial of a new drug (Mason et al., 2007). The median of resting heart rate was $68(\mathrm{bpm})$ for women and $66(\mathrm{bpm})$ for men. In another study about heart rate and life expectancy, it has reported that a correlation exists between mammalian heart rate and lifespan (Levine, 1997). According to this study, animals with low heart rate such as elephants have a longer life expectancy, and small animals with higher heart rate such as rats report shorter life span. An epidemiological study that investigated the relationship between heart rate and mortality rate or the incidence of cardiovascular accidents, studied during an average 16.5 years 8800 men and women between 30 and 59 years old in 1980 (Okamura, 2002). This study showed the tendancy that both number of death and cardiovascular mortality were higher with higher heart rate. Thus, as the heart rate is lower, the risk of death decreases and the lifespan becomes longer, which was also evident in previous studies. However, according to data released by the Ministry of Health, Labor and Welfare, the average life expectancy as of 2015 was 80.79 years for men and 87.05 years for women (MHLW, 2015), such in view of the heart rate at rest in previous studies, we cannot explain women longevity.

\subsubsection{Gender Differences in Heart Rate in Daily Life}

The heart rate at rest measured in previous studies is greatly influenced by physical internal factors, but it is hard to say if the influence of external factors such as physical activity, climate, mental stress due to work, and such is taken into consideration. Therefore, in this research, we analyzed gender difference in heart rate from 10 million cases of heart rate data measured in daily life by using formerly described smartphone system (COCOLOLO). Figure 3 shows the average value of the heart rate of men and women. The results of the student's t test are shown in Table 2. As a result, the average value of men was $72.53(\mathrm{bpm})$, and the average value of women was 71.53 (bpm), women's heart rate tending to be significantly lower by about 1 beat per minute. This tendancy is in conflict with the gender difference of the heart rate at rest reported by previous studies.

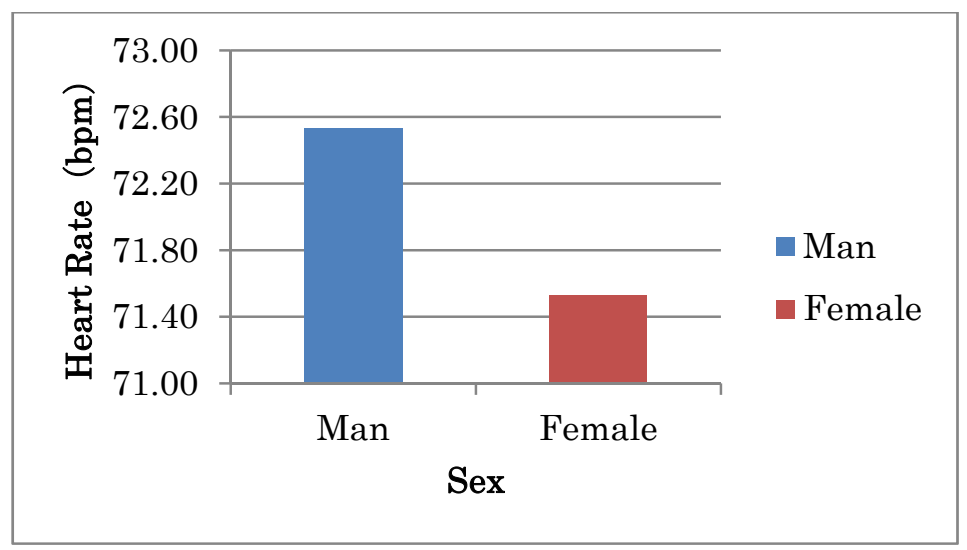

Figure 3. Relationship between heart rate and gender in daily life in this study 
Table 2. Result of student's t test (comparison of men and women heart rate)

\begin{tabular}{lllll}
\hline Gender & $N$ & Mean & Std. Deviation & Std. Error Mean \\
\hline Men & 3082010 & 72.53 & 12.335 & 0.007 \\
Women & 8361785 & 71.53 & 10.858 & 0.004 \\
\hline
\end{tabular}

\subsection{Relation Between Heart Rate and Age}

Next, we examined the relationship between heart rate and age. The previous study by Nakayama (1999), the resting heart rate measured during general medical examination on 8623 men and 4919 women, showed that in all ages men have a lower resting heart rate than in women (Nakayama, 1999). In the later study by Mason (2007), the age trend of resting heart rate for 46129 citizens who participated in clinical trials of new drugs were also analysed. The results confirmed previous tendancy that the resting heart rate of men in each age group tend to be lower by 2 to 4 beats per minute than the resting heart rate of women (Mason, 2007).

In this study, we analyzed relationship between heart rate and age from 10 million cases of heart rate data measured in daily life. We classified the data into six age groups from teenagers to 60 s and over, and we conducted multiple comparisons by the method of Games-Howell in order to investigate the difference between the age groups. The results are shown in Figure 4. As a result, when looking at the tendency by age, the heart rate of the woman tended to decrease as the age increased with the peak at $72.43(\mathrm{bpm})$ in the thirties. Meanwhile, men showed a tendency to increase in heart rate with a peak at $73.62(\mathrm{bpm})$ in the forties. The tendency by this age agrees roughly with the trend excluding the teenagers in the study by Mason (2007). We can consider that this trend of heart rate by age is not only influenced by internal factors such as aging, but also external factors such as work and physical activity, in which men may be more involved than women.

Previous studies have shown that stress caused by work contributes to increase blood pressure, accelerates the development of cardiovascular organ disorders such as cardiac hypertrophy, and is also related to the risk of coronary arrhythmia (Schnall, 1990, Tennant, 2000). Men have a tendency of heart rate being shaky after their 60s, which correspond to the age many people are retiring from work. Being in a situation that the stress from work is reduced, and the relaxation of mind can be kept easier, which possibly contribute to heart rate decline. In the present study, the heart rate of women showed a tendency significantly lower than that of men, specially from the 30 s, which correspond to the age when responsibilities at work became heavier. Thus, in terms of heart rate during daily life, this result may explain why women lived longer.

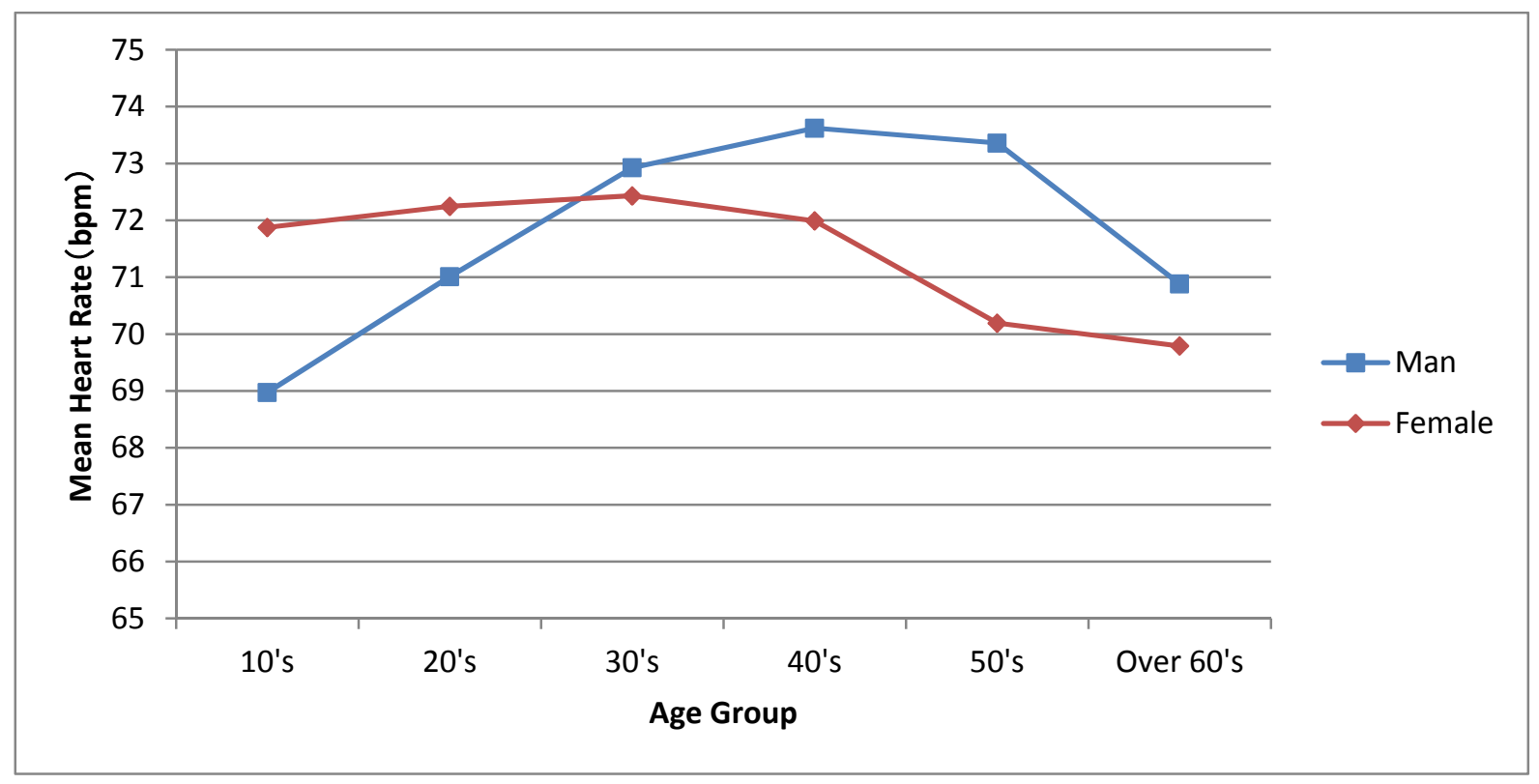

Figure 4. Relationship between heart rate and age in daily life in this study 


\subsection{Relationship Between Heart Rate and BMI}

In this section, we examined the relationship between heart rate and Body-Mass Index (BMI). BMI is the ratio of the weight $(\mathrm{kg})$ to the square of the height $(\mathrm{m})$, and represents the degree of obesity of a person. Moreover, the degree of obesity is calculated from BMI according to the criteria defined by the Japan Society for the Study of Obesity (Ogawa, 2015). In a previous study, Saito (1998) analyzed the relationship between resting heart rate and the degree of obesity for 4695 men and 1849 women aged from 18 to 22, who underwent routine physical examinations at college. The results confirmed that the obese persons tend to have a significantly higher heart rate compared with the non-obese persons (Saito, 1998).

In this study, we analyzed the relationship between heart rate and BMI, from 10 million cases of heart rate data measured in daily life. Here, we classified the degree of obesity from low body weight to obese into four groups, and we conducted multiple comparisons by the method of Games-Howell in order to investigate the difference between the groups. The results are shown in Figure 5. As the BMI increases, the heart rate tends to be significantly higher in both men and women. In addition, the heart rate tends to be the lowest in the case of the standard weight compared to the low body weight, and the heart rate of obese persons ( 2 degrees or more) is significantly higher by about 5 beats per minute than the heart rate of standard weight persons. Thus, the results of this study confirm the trend of previous studies.

In Framingham study, it is reported that the higher the heart rate, the higher the total death and the risk of death from cardiovascular events in high blood pressure patients (Gillman, 1993). In addition, lack of exercise increases the risk of various cardiovascular diseases, and it has been shown that there is a blood pressure lowering effect by moderate exercise habits (Rogers, 1996). Thus, it is reported that as obese people tend to have high blood pressure and high heart rate, the risk of various diseases increases, such a good balance of dietary habits and regular physical exercises habit help keeping a standard weight, which is very important for diseases prevention and healthcare.

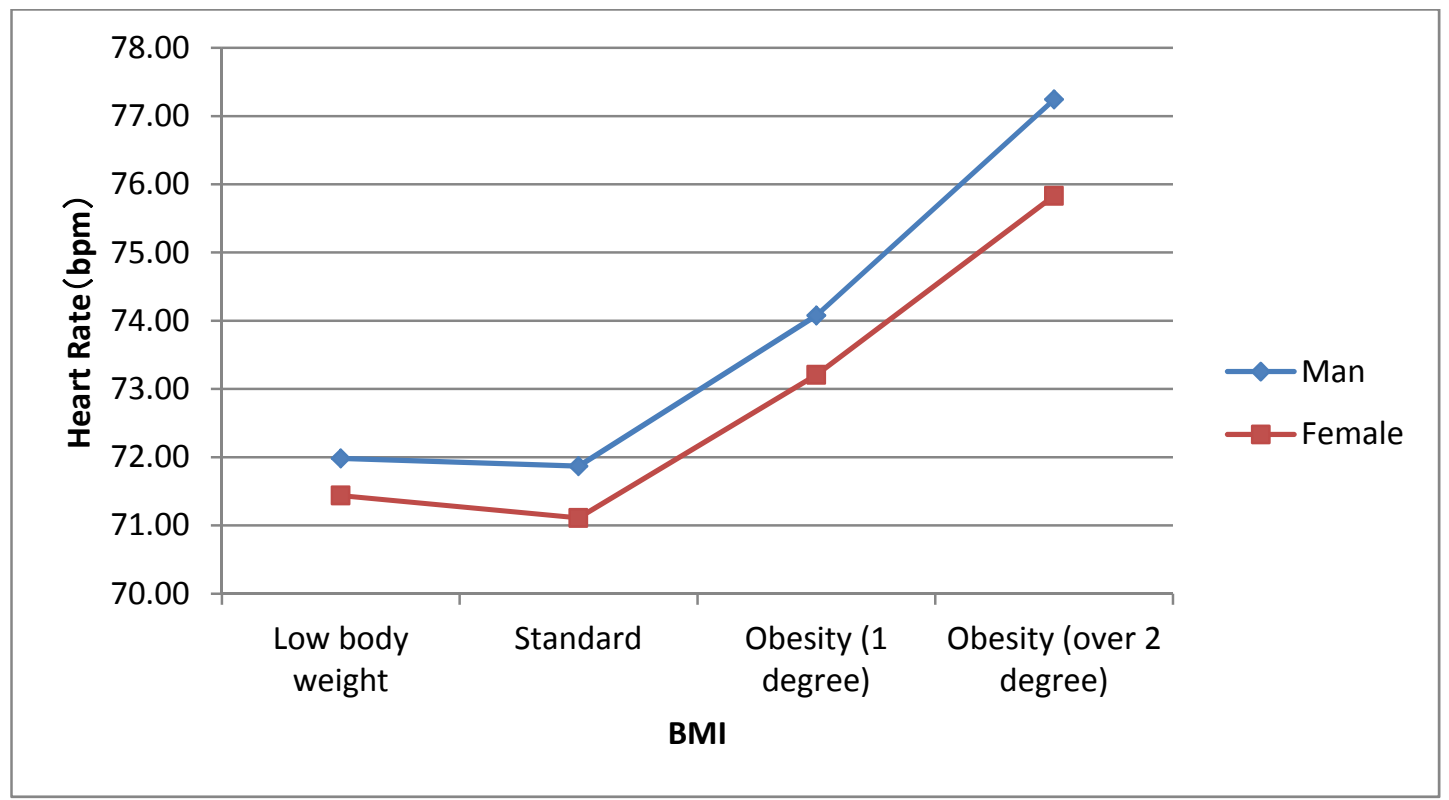

Figure 5. Relationship between resting heart rate and non obesity, obesity in this study

\subsection{Daily Fluctuation of Heart Rate}

The diurnal variation, also called circadian rhythm, is a mechanism that oscillates with a 24-hour cycle. It is known that the fundamental function of the body such as body temperature and hormone secretions also show a rhythm of about 24 hours. Circadian rhythm also discernable even in a condition where there is no change in light or temperature, so it is clear that the organism has a clock mechanism in the body, called the body clock. A previous study, which carried out a constant routine experiment on 8 healthy men kept in lying position and arousal for more than 24 hours, has shown that there is a circadian rhythm in resting heart rate under constant conditions (Vandewale, 2007). It confirmed a circadian rhythm with the lowest heart rate was early in the morning, then rising 
from there, up to a peak appearing during the day, to finally decrease from the evening to the night.

In this study, we analyzed the daily fluctuation of heart rate from 10 million cases of heart rate data measured in daily life. In this case, we classified the measurement time into eight groups of 3 hours, and we conducted multiple comparisons by the method of Games-Howell in order to investigate the difference between the groups. The results are shown in Figure 6. Heart rate during everyday life was lowest between 3 and 6 a.m. as in previous study, rising from there to a maximum at 12 to 15 o'clock during the day, after which the heart rate drops as it goes from evening to night, confirming the presence of a circadian rhythm. The average maximum heart rate was higher by about 7 beats per minute in the daytime than at dawn.

It was reported in a previous study that the incidence of myocardial infarction from 6 a.m. to noon was 3 times more frequent than in the evening, corresponding to the time zone when the heart rate most increases according to our study (Muller, 1989). Thus, in helping being aware of his heart rate, we think our system may help in preventing such diseases.

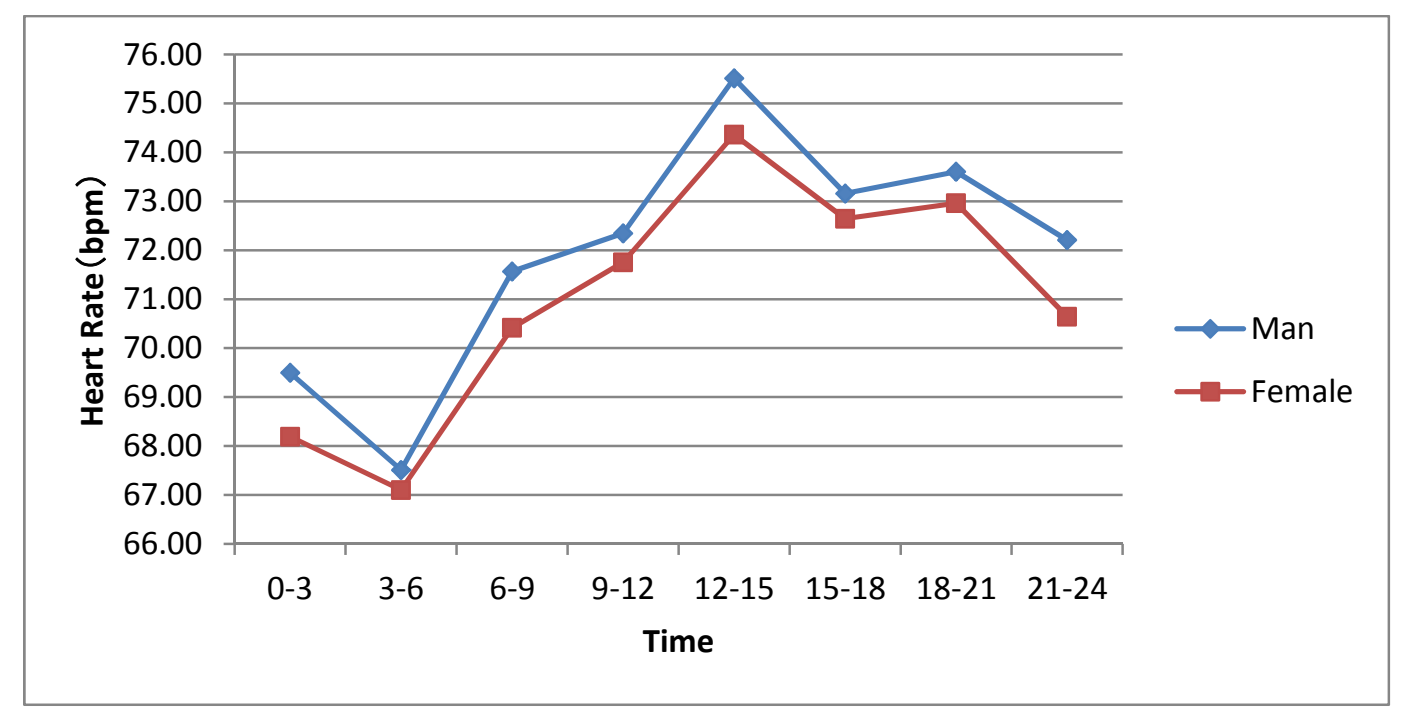

Figure 6. Daily fluctuation of resting heart rate in this study

\subsection{Weekly Fluctuation of Heart Rate}

In a previous study, a health check was conducted for the residents of certain towns and villages in Hokkaido to prevent the occurrence of cardiovascular disorders, and as one of the trials, 944 people aged 40 to 65 were targeted to analyze the trend of blood pressure between days of the week (Ohtsuka, 2011). This study revealed that the measured blood pressure value and daytime / nighttime blood pressure fluctuation differed depending on the day of the week. Blood pressure on Saturdays and Sundays showed a tendency to decrease significantly compared with blood pressure on weekdays. Specially, it had a tendency to rise rapidly on Monday after falling on weekends. These tendencies appeared more strongly by civil servants (employees), but also appeared significantly among farmers (self-employed persons).

In this study, we divided into seven groups for each day of the week, and we conducted multiple comparisons by the method of Games-Howell in order to investigate the difference between the groups. The results are shown in Figure 7. For both men and women, the heart rate tended to decrease on weekends and to rise on weekdays, which tendancy generally agrees with the weekly fluctuation in blood pressure of previous study. In particular, the difference in heart rate between Sunday and Monday is remarkable, heart rate on Mondays being $1.5 \mathrm{bpm}$ higher for men and $1 \mathrm{bpm}$ higher for women than on Sundays. We can reasonably assume that work stress may have a large impact. Therefore, among weekdays it is possible that the burden on the mind and body may be bigger on Mondays.

Concerning the relationship between the incidence of various diseases and the days of the week, the research group of Professor K. Kurafuri, analyzed the incidence of stroke for 12529 patients over the age of 40. The fluctuation due to the day of the week clearly showed a tendency regardless of age and gender of high occurrence rate on Monday, while being the lowest on Sunday (Kurashiki, 2004). In addition, in a survey conducted by the Osaka 
Acute Coronary Insufficiency Study (OACIS) group consisting of 25 centers, the examination of the number of occurrences by day of week for those who had an acute myocardial infarction during the four years from 1998 to 2001 revealed that myocardial infarction occurrs more frequently on Monday (Kinjo, 2003).

We can consider that the rapid rise in heart rate from Sunday to Monday, as shown in the present study, may contribute to the weekly fluctuation of the incidence of such disease. Also, the difference in heart rate between Sunday and Monday for men tends to be larger than for women. According to the multiobjective cohort study by the National Cancer Center, the incidence and the mortality rate of myocardial infarction and stroke tend to be higher in men than in women, and there is a possibility that there is a cause-and-effect relationship with the gender difference of the day-of-week variation shown in this study (NCC, 2010). So, it is very important to understand the weekly fluctuation of vital signs to prevent various diseases by for example lowering the burden of work on Monday morning.

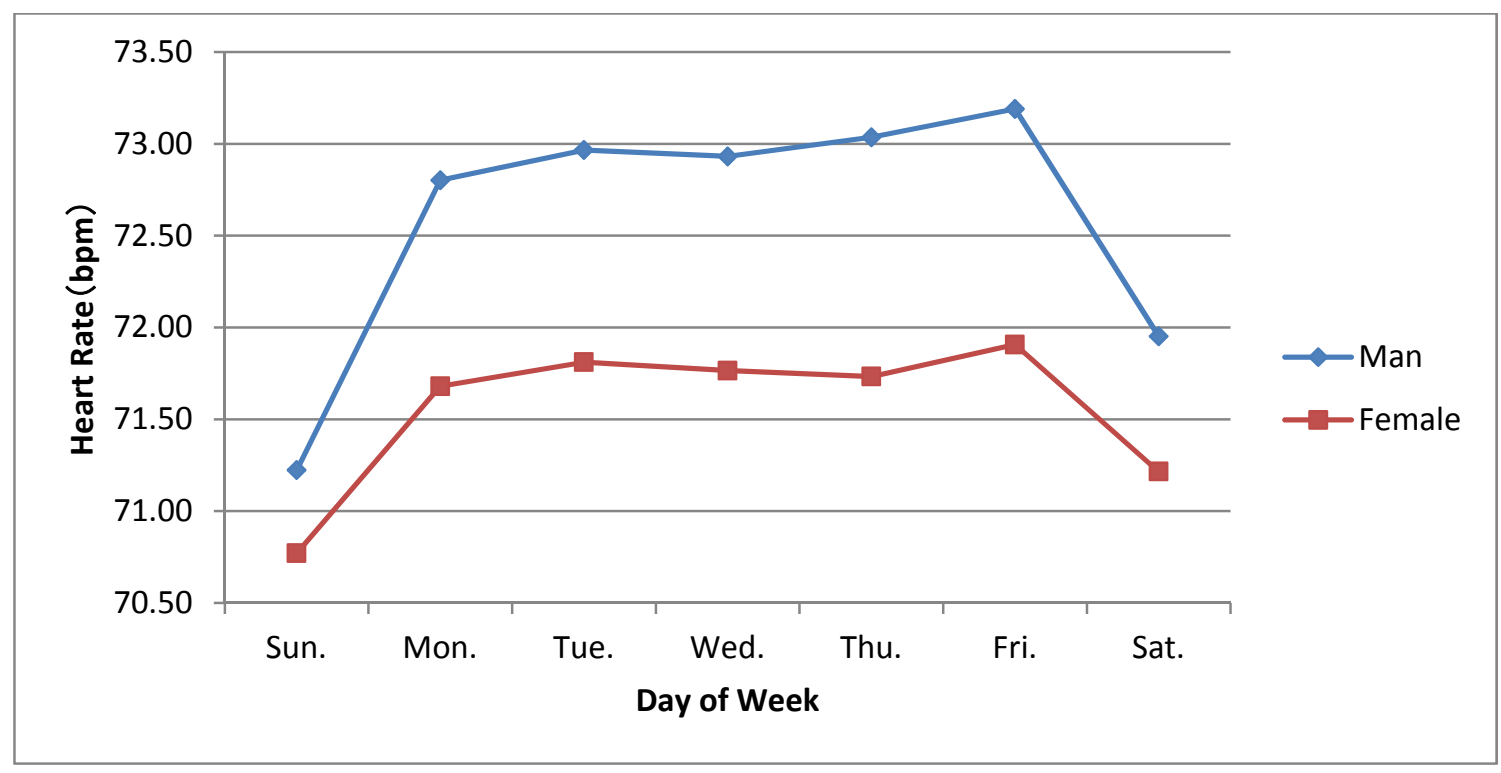

Figure 3. Weekly variation of heart rate during daily life in this study

\subsection{Seasonal Fluctuation of Heart Rate}

In this section, the seasonal variation of the heart rate was verified. Previous studies have investigated the relationship between blood pressure and seasonal fluctuation in the Sub-Arctic inhabitants. In that study, it is confirmed that blood pressure decreases in summer and increases in winter. It is thought to be a physiological phenomenon in which the blood pressure is elevated due to artery contraction when the temperature is low like in winter (Hopstock, 2013). Moreover, from the observation of blood pressure for every season in Japan, it is clear that blood pressure is generally low in summer and high in winter (Takahashi, 1955). In addition, according a study on hypertensive patients who measured blood pressure by home sphygmomanometer six times a day for five consecutive years while administering an antihypertensive treatment, the autumn showed the lowest trend and winter the highest (Maeda, 1993).

In this study, seasonal fluctuation of heart rate was analyzed from 10 million cases of heart rate data measured in daily life. We separated data for each month to create 12 groups, and conducted multiple comparisons by the method of Games-Howell in order to investigate the difference between the groups. The results are shown in Figure 8. The heart rate trend of both men and women showed a tendency to decline as summer approaches, until fall, to then increase in winter. We can assume that external factors such as climate comfort may be affecting the heart rate since it tends to be the lower in autumn than in summer. There is also a tendency common to men and women that the heart rate has a difference of about $1.5 \mathrm{bpm}$ between itd maximum and minimum through the year. The tendency showed by our results is generally consistent with the seasonal fluctuation in blood pressure reported in previous studies.

In an investigation conducted by nationwide hospitals of the Japan Organization of Occupational Health and Safety, on 46031 patients hospitalized for stroke, it has been verified that cases of cerebral haemorrhage and subarachnoid 
hemorrhage, occur significantly more often in winter than in summer (Toyota, 2011). This seasonal difference may be related to the seasonal fluctuation of heart rate in daily life as shown in our study. So, understanding the seasonal variation of vital signs can be said to be very important in preventing various diseases.

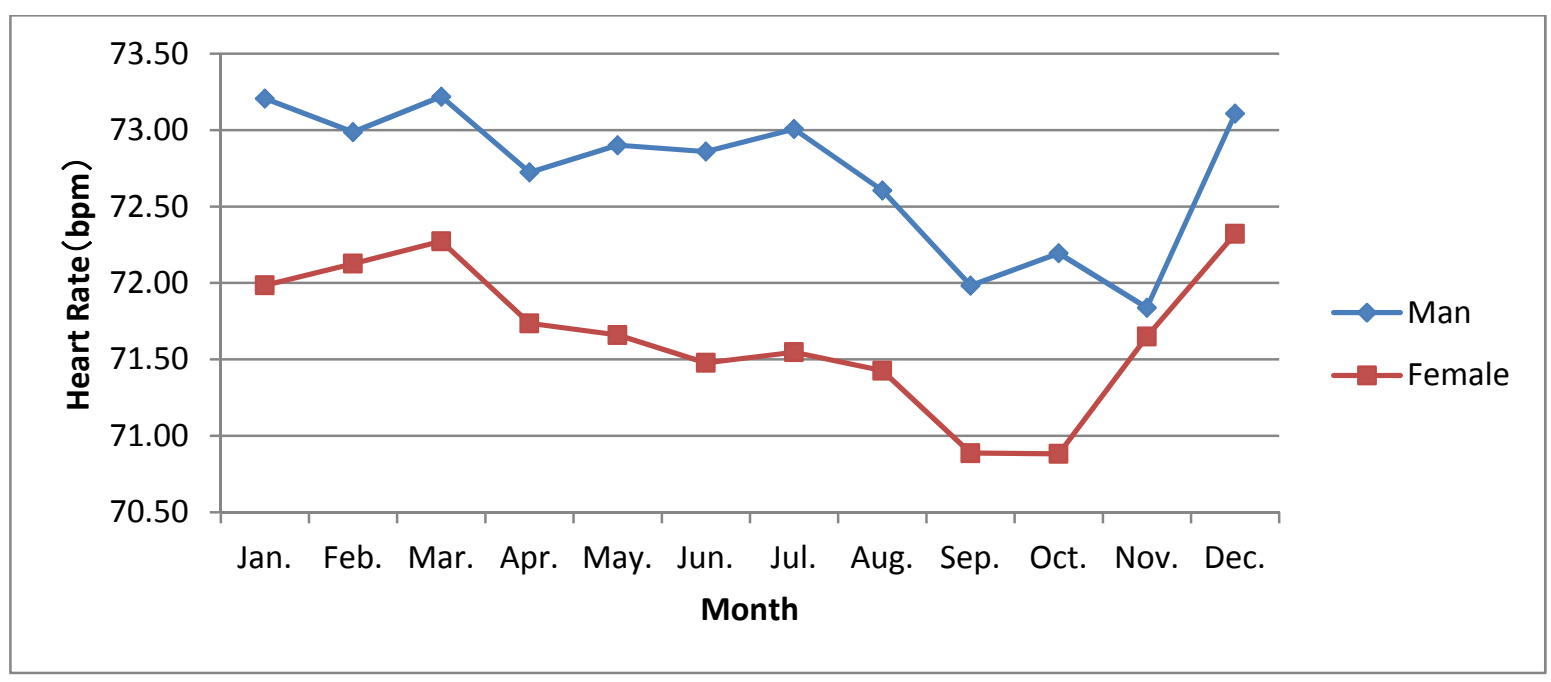

Figure 8. Seasonal variation of heart rate during daily life in this study

\section{Conclusion}

In this study, we attempted to analyze the trend of heart rate during people's daily life based on heart rate big data (10 million measures on nearly 1 million people). As a result, the following points were clarified.

- In the relationship between heart rate and gender, women tend to have a significantly lower heart rate than men. In the relationship between heart rate and age, heart rate is decreasing as age increases forn women, while it tends to rise from 40 to 50 years old for men. Up to their $20 \mathrm{~s}$, men have lower heart rate than women, though from the $30 \mathrm{~s}$ and later women then tend to have lower heart rate than men. These results may explain the reason why women live longer could be explained from the viewpoint of heart rate during daily life.

- In its relationship with BMI, heart rate tends to increase significantly as BMI increases, showing the importance of balanced diet, and regular physical activity.

- Concerning the relation between heart rate and age, our study revealed some differences with previous studies. While it has been reported that women tend to have higher average heart rate than men in all ages, our study showed a tendency that average heart rate of men was higher than women's after 30's. This result suggests that average heart rate in daily life may be an explanation of the reason why women tend to live longer than men.

- Regarding the daily fluctuation of the heart rate, the time interval between $3-6$ a.m. shows the lowest heart rate, then rising to reach its maximum between $12-15$ o'clock, to finally decrease as it goes from evening to night, confirming a circadian rhythm.

- As for the weekly fluctuation of the heart rate, it suddenly decreases on the weekend to be higher on weekdays. Especially the rise in heart rate from Sunday to Monday is remarkable.

- Regarding the seasonal fluctuation of the heart rate, it decreased from summer to autumn to take its maximum in winter.

The results of this research tend to be roughly in agreement with the results in previous research measured by dedicated equipment, in restricted conditions, and on a smaller or less sample number. It was possible to show the reliability and validity of the data measured in this system. From the viewpoint of public health, it can be said that it is important to compare and analyze people's health distribution according different attributes and spatiotemporal spaces. We believe that the findings obtained in this study can be used by the whole society as an indicator for health management and prevention of various diseases. 


\section{Acknowledgements}

The authors would like to thanks Kiyoshi Itao (professor emeritus, The University of Tokyo) for his numerous advices and suggestions all through this research work.

\section{Competing Interests Statement}

The authors declare that there are no competing or potential conflicts of interest.

\section{References}

Gillman, M. W., Kannel, W. B., Belanger, A., \& D'Agostino, R. B. (1993). Influence of heart rate on mortality among persons with hypertension: the Framingham Study. American heart journal, 125(4), 1148-1154. https://doi.org/10.1016/0002-8703(93)90128-V

Hopstock, L. A., Barnett, A. G., Bønaa, K. H., Mannsverk, J., Njølstad, I., \& Wilsgaard, T. (2013). Seasonal variation in cardiovascular disease risk factors in a subarctic population: the Tromsø Study 1979-2008. $J$ Epidemiol Community Health, 67(2), 113-118. https://doi.org/10.1136/jech-2012-201547

Japan Ministry of Health, Labour and Welfare (2010). Summary Report of Comprehensive Survey of Living Conditions 2010 - III-3 Worries and Stress. Retrieved from http://www.mhlw.go.jp/english/database/db-hss/dl/report_gaikyo_2010.pdf (2017/5/29)

Japan Ministry of Health, Labour and Welfare (2015). The 22nd Life Tables. Retrieved from http://www.mhlw.go.jp/english/database/db-hw/lifetb22nd/dl/tables.pdf

Japan National Cancer Center (2010). Japan Public Health Center-based Prospective Study (JPHC Study). Retrieved from http://epi.ncc.go.jp/en/jphc/index.html (2017/5/29). [in Japanese]

Karasudani, A., Nakano, Y., \& Mori, S. (2012). Heart Rate Monitor with a User Feedback Function Using a Cellphone Camera. IPSJ Transactions on Consumer Device and System, 2(1), 38-47. [in Japanese]

Kinjo, K., Sato, H., Shiotani, I., Kurotobi, T., Ohnishi, Y., Hishida, E., ... \& Fukunami, M. (2003). Variation during the week in the incidence of acute myocardial infarction: increased risk for Japanese women on Saturdays. Heart, 89(4), 398-403. https://doi.org/10.1136/heart.89.4.398

Kitani, T. (2000). Study research work report about the fatigue reduction technique for weary actual condition survey and positive health: Public welfare scientific research fund subsidy healthy scientific general research business, Fiscal Year 2000 report. [in Japanese]

Komazawa, M., Itao, K., Kobayashi, H., \& Luo, Z. (2016). Measurement and Evaluation of the Autonomic Nervous Function in Daily Life. Health, 8(10), 959-70. https://doi.org/10.4236/health.2016.810099

Kurashiki, K. (2004). Seasonal and Weekly Variation of Stroke Occurrence in Tottori Prefecture. $J$ Yonago Med Ass, 55(5), 249-257. [in Japanese]

Levine, H. J. (1997). Rest heart rate and life expectancy. J Am Coll Cardiol, 30, 1104-06.

Maeda, T., et al. (1993). Analysis of Daily, Weekly, and Seasonal Variations of Home Blood Pressure Measured Consecutively for 5 Years. Japanese circulation journal, 57(Supplement III), 1012. [in Japanese]

Mason, J. W., Ramseth, D. J., Chanter, D. O., Moon, T. E., Goodman, D. B., \& Mendzelevski, B. (2007). Electrocardiographic reference ranges derived from 79,743 ambulatory subjects. Journal of electrocardiology, 40(3), 228-234. https://doi.org/10.1016/j.jelectrocard.2006.09.003

Muller, J. E., Tofler, G. H., \& Stone, P. H. (1989). Circadian variation and triggers of onset of acute cardiovascular disease. Circulation, 79, 733-743. https://doi.org/10.1161/01.CIR.79.4.733

Nakayama, E., et al. (1999). A Study on the Heart Rate of Healthy Subjects in the General Health Examination. Jpn J Physiol Anthropol: JJPA, 4(2), 103-6. [in Japanese]

Ogawa, W., \& Miyazaki, S. (2015). Diagnosis criteria for obesity and obesity disease. Health Evaluation and Promotion: HEP, 42(2), 59-64. [in Japanese] https://doi.org/10.7143/jhep.42.301

Okamura, T., Hayakawa, T., Kadowaki, T., Kita, Y., Okayama, A., Elliott, P., ... \& NIPPONDATA80 Research Group. (2004). Resting heart rate and cause-specific death in a 16.5-year cohort study of the Japanese general population. American heart journal, 147(6), 1024-1032. https://doi.org/10.1016/j.ahj.2003.12.020

Ohtsuka, K. (2011). Blood Pressure Long-term Monitoring and Physiological Rhythm. Heart \& Wellness, 9, 1-6. [in Japanese] 
Rogers, M.W., et al. (1996). Differential effects of exercise training intensity on blood pressure and cardiovascular responses to stress in borderline hypertensive humans. $J$ Hypertens., 14(11), 1369-75. https://doi.org/10.1097/00004872-199611000-00017

Sagie, A., Larson, M. G., Goldberg, R. J., Bengtson, J. R., \& Levy, D. (1992). An improved method for adjusting the QT interval for heart rate (the Framingham Heart Study). Am $J$ Cardiol, 70, 797-801. https://doi.org/10.1016/0002-9149(92)90562-D

Schnall, P. L., Pieper, C., Schwartz, J. E., Karasek, R. A., Schlussel, Y., Devereux, R. B., ... \& Pickering, T. G. (1990). The relationship between'job strain,'workplace diastolic blood pressure, and left ventricular mass index: results of a case-control study. Jama, 263(14), 1929-1935. https://doi.org/10.1001/jama.1990.03440140055031

Singh, J., Larson, M. G., O'Donnell, C. J., Tsuji, H., \& Levy, D. (1998). Heritability of heart rate variability: the Framingham heart study. Journal of the American College of Cardiology, 31, 169. https://doi.org/10.1161/01.CIR.99.17.2251

Takahashi, E., Sasaki, N., Takeda, J., \& Ito, H. (1955). Observations on blood pressure in winter and summer of the same farmers in northeastern parts of Japan. Hirosaki Med. J, 6, 181-187. [in Japanese]

Tennant, C. (2000). Work stress and coronary heart disease. Journal of Cardiovascular Risk, 7(4), 273-76. https://doi.org/10.1177/204748730000700405

Toyoda, A. (2011). Seasonal variation in stroke incidence in Japan: 46,000 cases in Rosai Hospitals' registries, 2002-2008. The Japan Stroke Society, 33(2), 226-235. [in Japanese] https://doi.org/10.3995/jstroke.33.226

Vandewalle, G., Middleton, B., Rajaratnam, S. M., Stone, B. M., Thorleifsdottir, B., Arendt, J., \& DIJK, D. J. (2007). Robust circadian rhythm in heart rate and its variability: influence of exogenous melatonin and photoperiod. Journal of sleep research, 16(2), 148-155.

WINFrontier Corporation. COCOLOLO - Checking 8 types of feeling from heart beat fluctuations. Retrieved from https://itunes.apple.com/jp/app/cocololo-cafe-kimochishea/id973325431?mt=8\&ign-mpt=uo\%3D4 and https://play.google.com/store/apps/details?id=com.winfrontier.COCOLOLO\&hl=ja (2017/5/29) [in Japanese]

Yamashina, A. (2011). HEART's Selection. Shinzo, 43(11), 1389 -1390.

\section{Copyrights}

Copyright for this article is retained by the author(s), with first publication rights granted to the journal.

This is an open-access article distributed under the terms and conditions of the Creative Commons Attribution license (http://creativecommons.org/licenses/by/4.0/). 